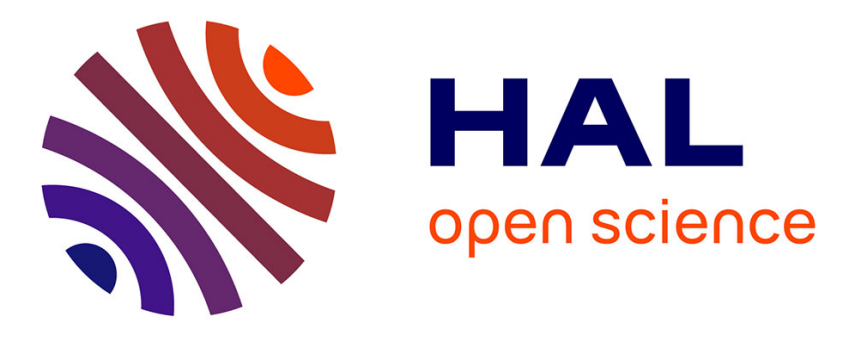

\title{
Hydrométrie et hydrologie historiques du bassin de la Durance
}

\author{
A. Kuentz, T. Mathevet, D. Coeur, C. Perret, J. Gailhard, L. Guérin, Y. \\ Gash, Vazken Andréassian
}

\section{- To cite this version:}

A. Kuentz, T. Mathevet, D. Coeur, C. Perret, J. Gailhard, et al.. Hydrométrie et hydrologie historiques du bassin de la Durance. La Houille Blanche - Revue internationale de l'eau, 2014, 4, pp.57-63. 10.1051/lhb/2014039 . hal-01128611

\section{HAL Id: hal-01128611 https://hal.science/hal-01128611}

Submitted on 10 Mar 2015

HAL is a multi-disciplinary open access archive for the deposit and dissemination of scientific research documents, whether they are published or not. The documents may come from teaching and research institutions in France or abroad, or from public or private research centers.
L'archive ouverte pluridisciplinaire HAL, est destinée au dépôt et à la diffusion de documents scientifiques de niveau recherche, publiés ou non, émanant des établissements d'enseignement et de recherche français ou étrangers, des laboratoires publics ou privés. 


\title{
Hydrométrie et hydrologie historiques du bassin de la Durance
}

\author{
Anna KUENTZ ${ }^{1,2}$, Thibault MATHEVET ${ }^{1}$, Denis CEUR ${ }^{2}$, Christian PERRET ${ }^{1}$, Joël GAILHARD ${ }^{1}$, \\ Laura GUÉRIN ${ }^{*}$, Yasin GASH ${ }^{*}$, Vazken ANDRÉASSIAN ${ }^{3}$
}

\author{
EDF-DTG, 21 avenue de l'Europe, 38040 Grenoble Cedex 9, anna.kuentz@gmail.com, thibault.mathevet@edf.fr \\ ACTHYS Diffusion, 253 chemin de Plate-Rousset, 38330 Biviers, denis.coeur@wanadoo.fr \\ 3. IRSTEA, 1 rue Pierre-Gilles de Gennes CS 100 30,92761 Antony cedex, vazken.andreassian@irstea.fr \\ * Étudiants en stage à EDF-DTG en 2012
}

\begin{abstract}
RÉSUMÉ. - Cette communication présente différents travaux que nous avons menés sur le bassin de la Durance. Ces travaux explorent l'incroyable patrimoine de données historiques en hydrologie et en hydrométrie de la Durance, où il a existé plus de 150 stations hydrométriques, avec un total de 4500 années de débits. Des séries de débits commençant au début du 20ème siècle ont pu être retrouvées, ainsi que de très nombreuses informations sur les stations hydrométriques, les techniques de jaugeages, les courbes de tarage, etc. Ce patrimoine historique met en évidence le très fort investissement des personnels pour développer les techniques de mesure et faire progresser la connaissance des ressources en eau des bassins. Ensuite, nous présentons des travaux que nous avons menés pour caractériser une partie de l'incertitude des données anciennes, liée au mode d'échantillonnage des hauteurs. Ces travaux montrent que les longues séries ne sont généralement pas homogènes d'un point de vue de la mesure et que les séries anciennes peuvent être significativement biaisées pendant la période de fonte. Enfin, nous présentons des travaux sur la caractérisation de la variabilité hydrologique du bassin de la Durance au cours des 100 dernières années. Ces travaux confirment l'intérêt de ces séries séculaires pour mieux connaître le fonctionnement des bassins versants.
\end{abstract}

Mots-clés : données historiques, validation, bassin versant, hydrométrie

\section{Historical hydrometry and hydrology of the Durance river watershed}

\begin{abstract}
This paper presents different studies on the Durance river watershed, situated in the south part of the Alps. Our studies explore a large collection of historical archives in hydrology and hydrometry, found by a historian. This watershed is characterised by an incredible density of hydrological stations, with more than 150 stations and 4500 years of hydrological records, since the beginning of the $20^{\text {th }}$ century. As an example, the archives of 10 stations starting between 1900 and 1917 were very detailed, with all the gaugings, rating curves, and streamflows records. Archives also reveal the great scientific and technical involvement at that period to understand, develop streamflow measurements and estimate available water resources. Then, we present an estimation of streamflow uncertainty of historical series due to the height measurement strategy. Results show that long streamflow series are not homogeneous in time and that old streamflows series could be significantly biased during snowmelt. Finally, this paper shows the great interest of historical time-series to better characterise hydrometeorological variability.
\end{abstract}

Key-words: historical data, validation, catchment basin, hydrometry

\section{INTRODUCTION}

La compréhension du fonctionnement du bassin de la Durance et de son évolution spatiale et temporelle est particulièrement intéressante pour EDF et l'ensemble des acteurs de l'eau. En effet, ce bassin, caractérisé par une grande diversité de régimes hydrologiques et d'importants enjeux liés à l'eau, fait l'objet de nombreux travaux de recherche, dont ceux du projet R2D2 "Risques, ressource en eau et gestion durable de la Durance à l'horizon $2050 »$ (https:// r2d2-2050.cemagref.fr/). Ces travaux visent notamment à analyser la sensibilité du fonctionnement naturel du bassin et des usages aux variabilités climatiques passées et futures.

C'est dans ce cadre que nous nous sommes intéressés à l'hydrométrie et l'hydrologie historiques de ce bassin. En effet, la Durance est connue pour son hydrométrie [Lallement, 2002] et son hydrologie historiques [Imbeaux, 1892] et de nombreuses sources documentaires témoignent de réseaux d'observation implantés depuis la fin du $19^{\text {ème }}$ siècle. Les recherches de Cœur [2012] dans différents fonds documentaires ont révélé l'extraordinaire patrimoine de ce bassin.

L'objectif de cet article est de présenter une synthèse de nos travaux autour de la thématique de l'hydrométrie et l'hydrologie historiques de la Durance. Nous présentons tout d'abord le patrimoine de données historiques, qui nous renseigne autant sur l'hydrologie que l'hydrométrie et les préoccupations techniques du début du $20^{\text {ème }}$ siècle. Ensuite, nous présentons des travaux sur la quantification de certaines des incertitudes des données anciennes, notamment l'homogénéité dans le temps de ces séries de données. Enfin, nous concluons sur l'intérêt de la valorisation des données anciennes pour caractériser la variabilité hydrologique d'un bassin versant. Cette thématique d'actualité renforce l'intérêt d'une valorisation des données historiques qui restent des témoins précieux du fonctionnement passé des bassins versants. 


\section{UN LARGE PATRIMOINE DE DONNÉES HISTORIQUES}

Les recherches de Cour [2012] ont eu pour objectif de recenser et de valoriser le patrimoine historique en hydrométrie et en hydrologie de la Durance de la fin du $19^{\text {ème }}$ et du début du $20^{\text {ème }}$ siècle (jusqu'en 1960). En effet, les premières mesures de débit à la station du Pont de Mirabeau datent de la première moitié du $19^{\text {ème }}$ siècle et plusieurs dizaines de stations hydrologiques ont été installées sur l'ensemble du bassin dès le début du $20^{\text {ème }}$ siècle. Les recensements effectués dans différentes sources (SHF, archives départementales et nationales, École Nationale des Ponts et Chaussées, DREAL PACA, SPC Grand Delta, EDF-DTG) ont permis de mettre au jour de nombreux documents relatifs à l'hydrométrie et l'hydrologie de ce bassin. Nous nous sommes concentrés sur les stations ayant des chroniques d'enregistrements continus de 1904 à nos jours, en s'intéressant particulièrement aux hauteurs, débits, jaugeages, courbes de tarage, localisation et vie de la station, conditions de mesure des hauteurs et techniques de jaugeages utilisées. Nous avons concentré nos analyses sur les stations de la Durance à la Vachette, Briançon, la Clapière et Pont de Mirabeau, l'Ubaye à Barcelonnette, le Buëch à Serres et aux Chambons, le Verdon à Colmars, l'Asse à la Clue de Chabrière. Ces documents constituent un très large patrimoine de données que nous avons partiellement analysé.

On retrouve tout d'abord dans ces documents l'existence d'un grand nombre de stations hydrométriques (plus de 150 stations) sur l'ensemble du bassin de la Durance, dont les mesures de hauteurs et de débits commencent pour certaines dès le début du $20^{\text {ème }}$ siècle et couvrent jusqu'à plus de 100 ans de mesures (Figure 1). Ces données représentent environ l'équivalent de 4500 ans de débits. Cette densité de mesures dès le début du $20^{\text {ème }}$ siècle apparait extraordinaire et témoigne d'une grande volonté de connaître le fonctionnement hydrologique de ces bassins, afin de se protéger contre les crues exceptionnelles suite aux évènements du milieu du $19^{\text {ème }}$ siècle (création en 1876 du Service Hydrométrique et d'Annonce des Crues du Rhône et de ses affluents, SHAC) et de prospecter le potentiel hydraulique de cette région. Nous avons

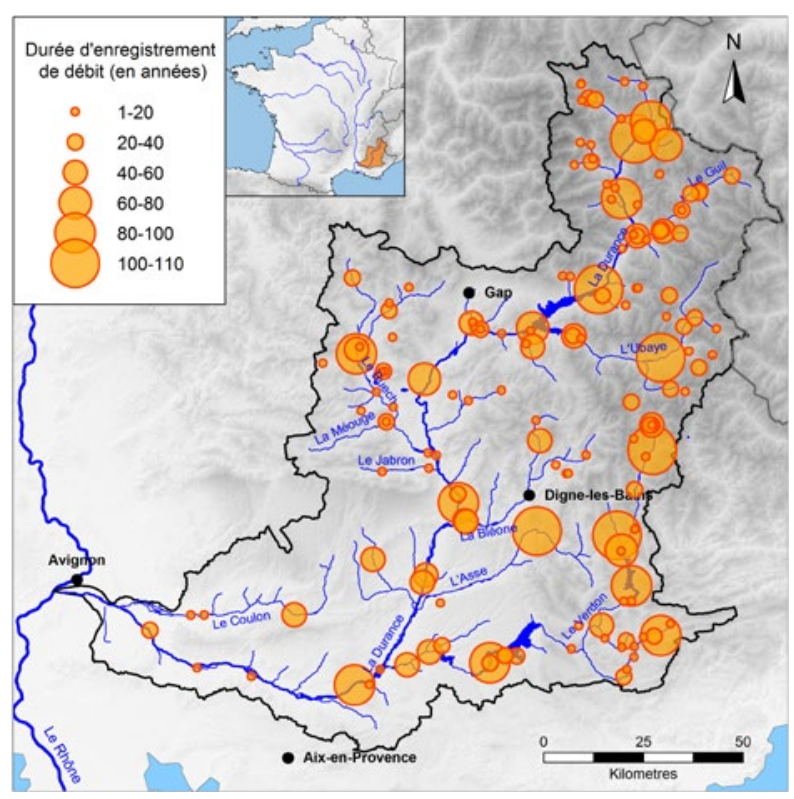

Figure 1 : Localisation et durée d'enregistrement des stations hydrométriques sur la Durance. également retrouvé des données relatives aux régimes des hauteurs d'eau établis dès la fin du $19^{\text {ème }}$ siècle à partir de mesures journalières (l'Ubaye à Barcelonnette, le Verdon à Colmars), sans pouvoir retrouver les séries journalières. Sur les 8 stations examinées en détail, nous avons retrouvé un total d'environ 1500 jaugeages et 150 courbes de tarage effectués avant 1960 . Les jaugeages étaient principalement réalisés par exploration du champ des vitesses et par la méthode de la dilution, apparue à partir des années 1950 aux stations de la Vachette et Colmars.

Les documents retrouvés témoignent d'une correspondance régulière entre les observateurs, chefs de stations, ingénieurs du bassin, ingénieurs en chef (Tableau 1). Ces documents montrent le fort investissement de l'ensemble du personnel pour le développement des matériels et techniques de mesures, des configurations des stations et d'un souci constant de la qualité des mesures. Ce fort investissement est sans doute la caractéristique essentielle de la mission des services hydrométriques, qui est de veiller en permanence à la qualité des observations, des mesures et aux progrès techniques dans le domaine. Cela concerne tous les champs d'activité, du plus théorique au plus concret et technique, en passant également par des considérations administratives et humaines. Les notions de progrès et d'innovations scientifiques et techniques sont ainsi de fait au cœur de la pratique des agents des services hydrométriques, sans forcément d'ailleurs qu'ils en aient eu toujours conscience. La diversité et la dynamique des conditions naturelles, les caractéristiques particulières des bassins de montagne avec un fort transport solide, ont également largement conditionné cette nécessité d'entretenir et d'adapter sans cesse les systèmes d'observation et de mesure, sans parler de l'intégration des progrès techniques eux-mêmes.

L'amélioration des stations se retrouve dans de nombreux documents qui témoignent de ces soucis permanents. Ces améliorations se sont concrétisées, au début du 20ème siècle, lors du passage de stations du SHAC d'observation des hauteurs et d'annonce des crues, à des stations hydrométriques avec une estimation quantitative des débits. Cette transformation, qui a eu lieu au moment de la mise en place du réseau des grandes forces hydrauliques, avait pour objectif de connaitre les régimes des débits sur l'ensemble du bassin tout au long de l'année (pas seulement pendant les crues). Ensuite, on retrouve également de nombreux documents sur les évolutions des matériels de la station : passerelles, treuils, limnigraphes, chariots de manœuvre, bateaux avec câble. Les constructeurs de matériels entretiennent des correspondances avec les ingénieurs du SHAC pour améliorer les techniques et matériels. La question de la sécurité est régulièrement évoquée pour faire progresser les conditions de la mesure, notamment sur les passerelles.

Ensuite, on retrouve de nombreux documents relatifs à la qualité des mesures de hauteurs. Concernant les mesures de hauteurs, la qualité de l'échelle, son emplacement, son accessibilité ou sa lisibilité sont souvent évoquées. La qualité de l'observateur, ses capacités à bien comprendre les instructions de lecture de l'échelle ou son assiduité sont parfois questionnées pour juger de la qualité d'estimations de débits. Des indications particulières sont données lorsque la rivière et l'échelle de mesure des hauteurs sont en glace.

Concernant les jaugeages, on retrouve de nombreux documents relatifs aux matériels utilisés : flotteurs (très rarement), tube de Pitot (ponctuellement pour les étiages), plus couramment les moulinets de Woltman, le moulinet Richard à deux hélices ou les moulinets Ott (nombreuses références différentes). A partir du début des années 1950, on retrouve l'apparition des jaugeages par dilution chimique sur la station de Colmars et la Vachette. Pour chaque station, on est capable 
Tableau 1 : Extrait des commentaires et correspondances retrouvées dans les archives des stations hydrologiques.

\begin{tabular}{|c|c|c|}
\hline Thématique & $\begin{array}{c}\text { Station } \\
\text { de jaugeage }\end{array}$ & Commentaire et date \\
\hline Matériel & $\begin{array}{l}\text { La Durance } \\
\text { à la Vachette }\end{array}$ & $\begin{array}{l}\text { "Nous avons ramené à Grenoble pour vérification et tarage le moulinet de M. Jarrel, } \\
\text { chef de station. Cet instrument n'avait pas été étalonné depuis } 1931 \text { " } \\
\text { (Inspection de la station par les GFH, 11/1945) }\end{array}$ \\
\hline Matériel & $\begin{array}{l}\text { L'Ubaye à } \\
\text { Barcelonnette }\end{array}$ & $\begin{array}{l}\text { "Passerelle légère, constituée par } 2 \text { à } 3 \text { échelles recouvertes de madriers et soutenues du } \\
\text { pont par des cordes amarrées aux poutres. Médiocre et peu sûre, à améliorer» (21/06/1908) }\end{array}$ \\
\hline $\begin{array}{l}\text { Matériel/ } \\
\text { Hauteur }\end{array}$ & $\begin{array}{l}\text { L'Asse } \\
\text { à la Clue } \\
\text { de Chabrière }\end{array}$ & $\begin{array}{l}\text { "La rigueur de la température s'est opposée pendant le premier trimestre à la mise en place } \\
\text { du treuil tracteur. Conformément aux instructions, la glace a été cassée tous les jours } \\
\text { par le cantonnier observateur qui débarrassait soigneusement les abords de l'échelle } \\
\text { avant de faire les lectures » (1 } 1^{\text {er }} \text { trimestre 1914) }\end{array}$ \\
\hline Hauteur & $\begin{array}{l}\text { L'Asse } \\
\text { à la Clue } \\
\text { de Chabrière }\end{array}$ & $\begin{array}{l}\text { "il est possible qu'au début l'observateur ne lisait pas correctement les cotes, } \\
\text { surtout les négatives. Je me rappelle fort bien le lui avoir expliqué » (15/06/1912) }\end{array}$ \\
\hline Jaugeage & $\begin{array}{l}\text { Le Buëch } \\
\text { aux } \\
\text { Chambons }\end{array}$ & $\begin{array}{l}\text { Concernant les jaugeages : "la difficulté de leur mise en place dû au régime torrentiel } \\
\text { de l'écoulement ainsi que de son irrégularité à l'emplacement du jaugeage " } \\
\text { "pour les basses eaux, les jaugeages sont effectués en aval en un point variable à chaque } \\
\text { opération, là où les eaux se trouvent le plus concentrées » (1909) }\end{array}$ \\
\hline Jaugeage & $\begin{array}{l}\text { Le Verdon } \\
\text { à Colmars }\end{array}$ & $\begin{array}{l}\text { "Il me parait utile, pour la sécurité des opérateurs, de pourvoir la passerelle d'un garde } \\
\text { corps à l'aval. On pourrait peut être se contenter d'un fort fil de fer tendu entre deux crochets } \\
\text { scellés au culées du pont. " } \\
\text { "Le Lit parait se maintenir fixe depuis } 1907 \text {; mais quelques gros blocs roulent à chaque } \\
\text { crue. Il est nécessaire de les enlever pour assurer dans le profil de jaugeage un écoulement } \\
\text { régulier sans lequel les mesures sont très imprécises " (22/08/1910) }\end{array}$ \\
\hline $\begin{array}{l}\text { Hauteur } \\
\text { et jaugeage }\end{array}$ & $\begin{array}{l}\text { La Durance } \\
\text { au Pont de } \\
\text { Mirabeau }\end{array}$ & $\begin{array}{l}\text { "les variations de débits ne correspondent pas aux hauteurs dreau lues à liéchelle ", } \\
\text { "la nécessité de faire des jaugeages à lıemplacement de l’échelle " (1901) }\end{array}$ \\
\hline $\begin{array}{l}\text { Période } \\
\text { de jaugeage }\end{array}$ & $\begin{array}{l}\text { La Durance } \\
\text { à la Vachette }\end{array}$ & $\begin{array}{l}\text { "un relâchement dans les périodes de transition, les eaux basses ne paraissent être observées } \\
\text { que de temps en temps " (1953) }\end{array}$ \\
\hline $\begin{array}{l}\text { Période } \\
\text { de jaugeage }\end{array}$ & $\begin{array}{l}\text { L'Asse } \\
\text { à la Clue } \\
\text { de Chabrière }\end{array}$ & $\begin{array}{l}\text { "La mauvaise saison étant passée, je prie M. Canton de réaliser sans plus tarder la mise en } \\
\text { place du nouveau treuil et de reprendre les opérations activement. Je rappelle que pour déterminer } \\
\text { la courbe de tarage, il manque plusieurs points à l'extrême étiage et aux crues » (22/04/1914) }\end{array}$ \\
\hline $\begin{array}{l}\text { Qualité } \\
\text { de la station }\end{array}$ & $\begin{array}{l}\text { L'Ubaye à } \\
\text { Barcelonnette }\end{array}$ & $\begin{array}{l}\text { "La station parait médiocre en raison de liinstabilité du lit et de limprécision des jaugeages. La } \\
\text { courbe de tarage est médiocre à tous les débits. La sensibilité de l’échelle est faible en basse eaux. " }\end{array}$ \\
\hline $\begin{array}{l}\text { Qualité } \\
\text { de la station }\end{array}$ & $\begin{array}{l}\text { L'Asse } \\
\text { à la Clue } \\
\text { de Chabrière }\end{array}$ & $\begin{array}{l}\text { "irrégularité du lit de la rivière où de gros blocs sont échoués dans le voisinage du profil } \\
\text { de jaugeage " (1944) }\end{array}$ \\
\hline $\begin{array}{l}\text { Qualité } \\
\text { des débits } \\
\text { et mesure } \\
\text { des hauteurs }\end{array}$ & $\begin{array}{l}\text { Le Verdon } \\
\text { à Colmars }\end{array}$ & $\begin{array}{l}\text { "Une étude comparative entre les différentes stations du bassin en } 1952 \text { signale des résultats } \\
\text { médiocres de débits à cette station. Ces erreurs auraient pour origine soit un détarage } \\
\text { du moulinet soit une erreur systématique de lecture à l'échelle. Il apparait par la suite } \\
\text { que l'échelle serait devenue illisible car n'ayant jamais était changée sur toute la période, } \\
\text { serait à l'origine de ces écarts " (1953) }\end{array}$ \\
\hline $\begin{array}{l}\text { Vie } \\
\text { de la station }\end{array}$ & $\begin{array}{l}\text { La Durance } \\
\text { à la Vachette }\end{array}$ & $\begin{array}{l}\text { "Le matériel de la station de jaugeage de la Vachette a été emporté ou détruit } \\
\text { par les allemands lors de leur retour à la Vachette au début septembre 1944" (24/11/1944) }\end{array}$ \\
\hline
\end{tabular}

de retrouver la liste des jaugeages effectués depuis leur création (environ 200 par station en 60 ans), les matériels utilisés, le dépouillement des verticales (parfois, avec la vérification des calculs et la validation du jaugeage par un supérieur). Comme pour la configuration de certaines stations, la dangerosité des jaugeages est évoquée. La question du choix de la bonne section de jaugeage (en fonction du débit et du lit de la rivière) est largement évoquée, les nombreux problèmes de transport solide et d'instabilité sont souvent évoquées (Verdon à Colmars, Asse à la Clue de Chabrière) pour regretter la difficulté de l'exercice et de l'obtention de données fiables de débits.

A partir des jaugeages à disposition, des courbes de tarages sont tracées et on en trouve de très nombreuses (10 à 20 par station en 60 ans). Encore une fois, de nombreuses correspondances et discussions portent sur la représentativité des courbes de tarage en fonction du régime des eaux (étiages, crue), des gammes trop limitées de débits jaugés ou de l'instabilité des sections de jaugeage et de mesure de hauteurs. Les difficultés climatiques sont rappelées pour dire que les périodes de jaugeages sont trop limitées dans le temps pour être capable de jauger les extrêmes de débits. Au-delà des considérations sur la qualité des jaugeages, des courbes de tarage et de la stabilité du fond des rivières, la qualité des débits était également estimée par comparaison graphique entre stations amont-aval ou par de simples modèles linéaires. Ces analyses permettaient d'incriminer les observateurs, les mesures de hauteurs, l'instabilité du lit des rivières ou la représentativité des courbes de tarages. 
Ces documents révèlent également les nombreuses inspections et tournées des ingénieurs des grandes forces hydrauliques. Ces inspections avaient pour objectif d'évaluer l'état physique et technique des stations de mesures, en pointant les améliorations à apporter au matériel (configuration de la passerelle, des limnigraphes, étalonnage des moulinets, etc.), la nécessité d'arrêter l'exploitation d'une station ou de déplacer une station (tout en conservant pendant une durée les deux stations afin d'assurer une période de recouvrement). Enfin, ces tournées servaient également à évaluer la qualité des données produites (mesures de hauteurs, jaugeages, courbes de tarage, débits), ainsi que la compétence et la disponibilité des agents.

\section{ANALYSE DE LA QUALITÉ DE LONGUES SÉRIES DE DEBITS}

\section{III.1. Mode de construction des séries historiques de débits}

L'analyse des données contenues dans les dossiersstations constitués lors des recherches décrites dans la partie précédente a permis de mettre en évidence, sur la plupart des stations, une succession de trois principales méthodes de construction de séries journalières de débits.

a. Au début du vingtième siècle, les séries étaient pour la plupart construite par lecture quotidienne à heure fixe de la hauteur d'eau à l'échelle : un observateur était chargé de lire chaque jour à la même heure la hauteur d'eau à l'échelle de la station. Cette hauteur était ensuite convertie en débit via une courbe de tarage et le débit obtenu était consigné comme débit journalier.

b. Des limnigraphes ont ensuite été mis en place petit à petit aux différentes stations. La hauteur d'eau était enregistrée graphiquement de manière continue grâce à cet appareil, et les limnigrammes ainsi obtenus étaient dépouillés manuellement pour en déduire une valeur de débit journalier. Plusieurs méthodes ont été utilisées pour le dépouillement des limnigrammes, principalement la moyenne des hauteurs " à vue " et la moyenne de $n$ valeurs de débits infrajournaliers (souvent $n=6$ ou $n=12$ ). Les dates d'installation des limnigraphes sont très variables d'une station à une autre, ainsi on en trouvait un dès 1904 à la station de Briançon sur la Durance, alors que la station de Colmars sur le Verdon n'en a été équipée qu'en 1958.

c. Actuellement, la hauteur d'eau est mesurée en continu, enregistrée et convertie en débit à un pas de temps horaire, le débit journalier est obtenu par moyenne des 24 valeurs horaires.

On voit bien par cette courte description que l'évolution des méthodes de construction des séries journalières de débits a été très importante au cours du siècle. Dans une même série de débit considérée à première vue comme continue, on trouve ainsi souvent une juxtaposition de séries de variables qui peuvent être différentes : le débit obtenu par la méthode (a) est par exemple un débit instantané à heure fixe, tandis que le débit obtenu par la méthode (c) est un débit moyen journalier.

Il paraît important d'analyser l'impact de cette évolution des méthodes sur la valeur finale des débits journaliers, puisqu'elle impacte potentiellement l'homogénéité des séries continues de débits, l'incertitude des données anciennes et les tests de tendance ou rupture que l'on peut faire sur de longues séries.

\section{III.2. Impact de la méthode de lecture quotidienne à l'échelle}

\section{III.2.1. Mode de comparaison}

Afin de quantifier l'impact de la méthode de lecture quotidienne à l'échelle sur la valeur finale du débit journalier, nous nous proposons de simuler cette méthode sur la période récente sur laquelle nous disposons de données horaires. Il sera ainsi possible de comparer le débit obtenu par la simulation de la méthode (a) à celui obtenu par la méthode (c), prise comme référence. Ainsi, pour chaque jour de la chronique simulée, l'erreur relative $\varepsilon_{\mathrm{j}, \mathrm{t}}$ entre le débit $\hat{\mathrm{Q}}_{\mathrm{j}, \mathrm{t}}$ obtenu par la simulation de la méthode (a) pour une heure de mesure $t$ donnée et le débit moyen journalier $\bar{Q}_{j}$ obtenu par la méthode (c) est calculée de la manière suivante :

$$
\varepsilon_{\mathrm{j}, \mathrm{t}}=\frac{\hat{\mathrm{Q}}_{\mathrm{j}, \mathrm{t}}-\overline{\mathrm{Q}}_{\mathrm{j}}}{\overline{\mathrm{Q}}_{\mathrm{j}}}
$$

\section{III.2.2. Analyse sur la station de Colmars}

A titre d'exemple, la Figure 2 représente, pour la période du printemps 2004 à la station du Verdon à Colmars, les séries de débit horaire, débit moyen journalier et débit journalier obtenu par simulation de la méthode (a) pour deux heures de lecture différentes. Dans la partie inférieure du graphique sont tracées les courbes de l'erreur relative journalière correspondant aux deux heures de mesure simulées.

Sur la courbe des débits horaires, on observe un cycle infra-journalier très marqué, lié aux variations infrajournalières de la fonte des neiges puis des glaciers. En raison de ce cycle, le débit moyen journalier peut être significativement éloigné du débit à une heure donnée de la journée. En effet, on peut constater par exemple que le débit correspondant à une lecture quotidienne à midi (comme c'était le cas à cette station avant 1958), est globalement situé sur l'enveloppe inférieure de la courbe des débits horaires. L'écart relatif $\varepsilon_{\mathrm{j}, \mathrm{t}}$ entre ce débit et le débit moyen journalier est donc globalement négatif, et peut atteindre $-30 \%$ du débit moyen journalier. En revanche, si la mesure quotidienne était faite à 5 heures plutôt qu'à midi, la valeur obtenue serait plus proche du débit moyen journalier, le biais est dans ce cas proche de zéro.

L'erreur relative $\varepsilon_{\mathrm{j}, \mathrm{t}}$ étant calculée pour chaque jour de la chronique simulée, on peut analyser statistiquement l'impact de la méthode (a) sur la valeur du débit journalier, et ce pour différentes périodes de l'année et différentes heures de mesure. La Figure 3 montre les distributions des erreurs $\varepsilon_{\mathrm{j}, \mathrm{t}}$ pour chaque mois de l'année et pour deux heures de lecture à l'échelle : $5 \mathrm{~h}$ du matin et midi. Ces deux graphes montrent que les propriétés statistiques de l'erreur relative entre débit moyen journalier et débit obtenu par simulation de la méthode de lecture quotidienne à l'échelle dépendent fortement de l'heure de mesure et de la période de l'année. Par ailleurs, on peut observer sur la Figure 3 que les distributions des erreurs pour le mois de mai sont très différentes selon l'heure de lecture. Pour une lecture à $5 \mathrm{~h}$, l'erreur moyenne du moi de mai est de $-1 \%$ environ, avec un intervalle interquantiles $10-90 \%$ allant de $-15 \%$ à $+12 \%$; Si la lecture est faite à midi, toujours pour le mois de mai l'erreur moyenne vaut $-9 \%$, et l'intervalle interquantiles $10-90 \%$ est beaucoup moins centré autour de zéro puisqu'il va de $-21 \%$ à $+4 \%$.

La répartition annuelle de l'erreur est également intéressante : que ce soit pour une lecture faite à $5 \mathrm{~h}$ ou à midi, 
Verdon@Colmars - 20/04/2004 - 10/06/2004

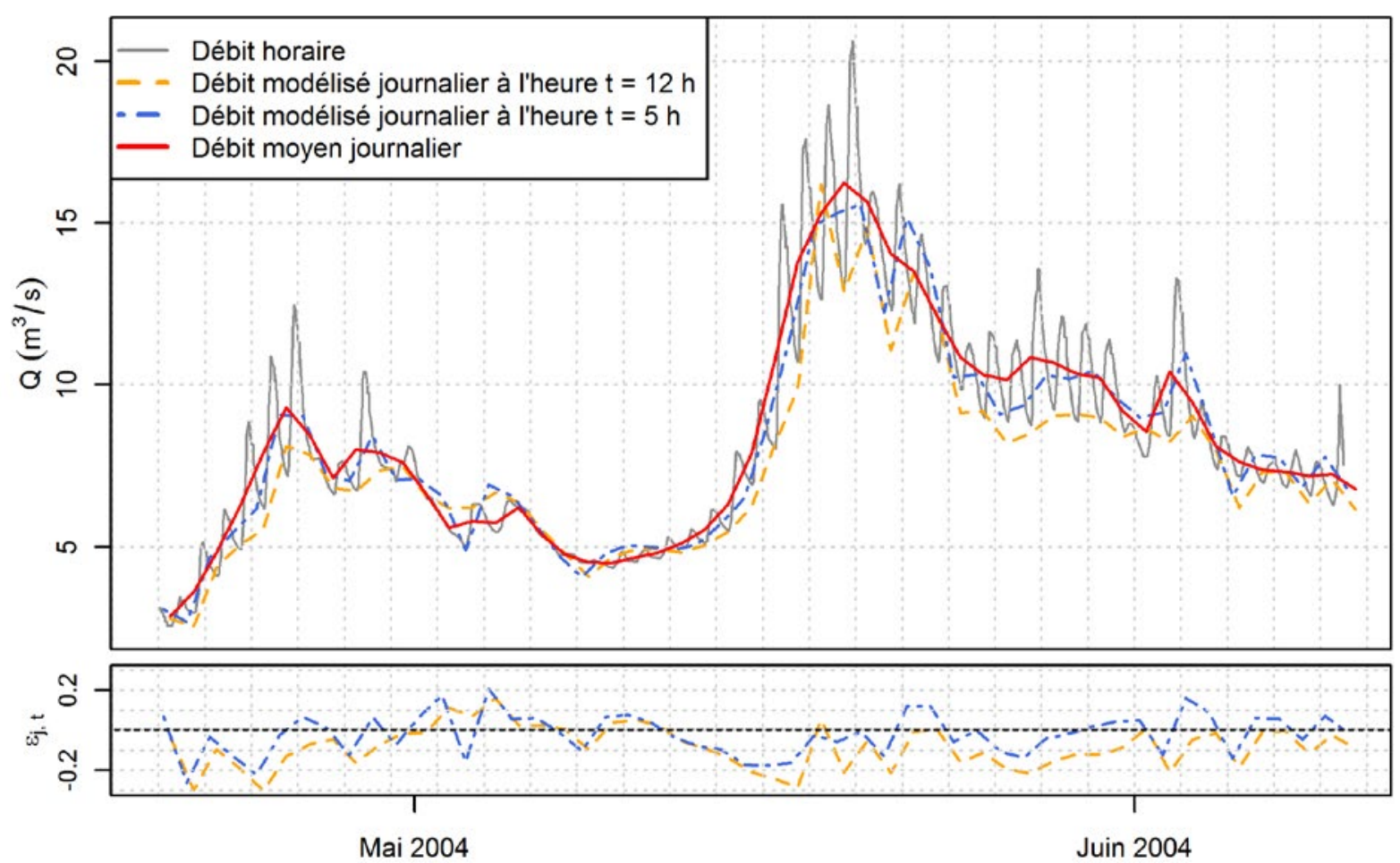

Figure 2: Évolution des débits horaires et journaliers moyens ou par lecture quotidienne à la Station de Colmars sur le Verdon, erreurs liées à la méthode de lecture quotidienne.

(a) Lecture ponctuelle à $5 \mathrm{~h}$

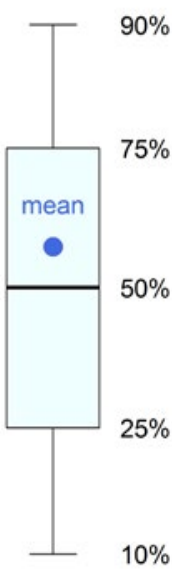

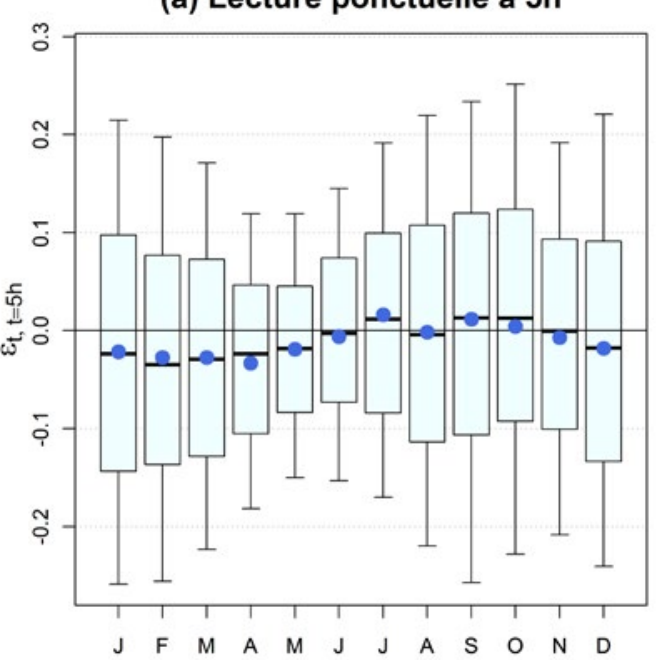

(b) Lecture ponctuelle à $12 \mathrm{~h}$

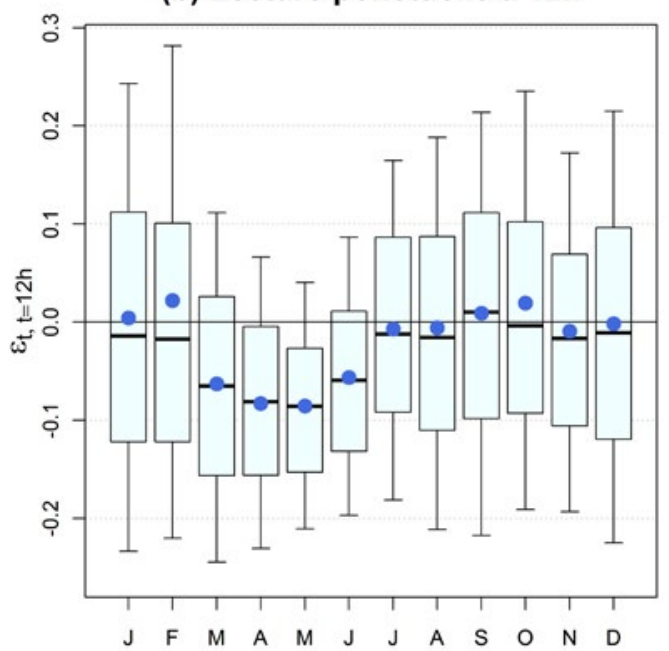

Figure 3 : Distributions mensuelles des erreurs relatives liées à la méthode de lecture ponctuelle à la station de Colmars sur le Verdon.

l'erreur relative est plus élevée pendant l'hiver et le printemps, saisons où les débits sont alimentés par la fonte des neiges. Des observations similaires ont pu être effectuées sur d'autres stations du bassin de la Durance, où l'on constate que l'impact de la méthode de lecture quotidienne de la hauteur à l'échelle est généralement plus fort sur les stations dont le régime possède une composante nivale. Par exemple, sur les bassins versant au régime nival, le biais sur le module peut atteindre 5 à $10 \%$.
III.2.3. Correction des séries construites par la méthode de lecture ponctuelle à l'échelle

Les observations précédentes ont permis de constater que la méthode de lecture quotidienne de la hauteur d'eau à l'échelle peut significativement biaiser le débit journalier, cela en fonction de l'heure de lecture et de la période de l'année. Afin de pouvoir homogénéiser les longues séries de débits dont une partie a été construite par cette méthode, 
nous proposons ici une méthode de correction de ce biais. Il s'agit simplement de s'appuyer sur le régime de l'erreur observée sur la simulation de la méthode sur la période récente de disponibilité de données horaires pour corriger la partie ancienne de la série.

A la station du Verdon à Colmars, la méthode de lecture quotidienne de la hauteur d'eau à l'échelle a été utilisée de 1904 à 1956, avec une lecture à midi. La Figure 4 montre, pour cette période, les régimes hydrologiques de la série d'origine, obtenue par lecture quotidienne à midi, de la série corrigée à l'aide de l'erreur moyenne mensuelle observée sur la période de simulation, et l'intervalle interquantiles $10-90 \%$ de correction.

Le régime hydrologique du Verdon à Colmars est de type nivo-pluvial, avec un maximum du débit moyen mensuel en mai-juin, et un maximum secondaire lié aux pluies d'automne en novembre. On remarque à nouveau le biais important lié au cycle de fonte pendant la période de hauts débits au printemps. L'intervalle interquantiles $10-90 \%$ est plus large pendant le printemps $(\sim 65 \mathrm{~mm} /$ mois en mai) et l'automne $(\sim 30 \mathrm{~mm} / \mathrm{mois}$ en novembre) qu'en hiver et en été. Cela peut s'expliquer par les crues qui ont principalement lieu pendant ces deux périodes et peuvent induire de forte variations infra-journalières de débit, sans qu'il n'y ait de systématisme sur l'heure du pic de crue.

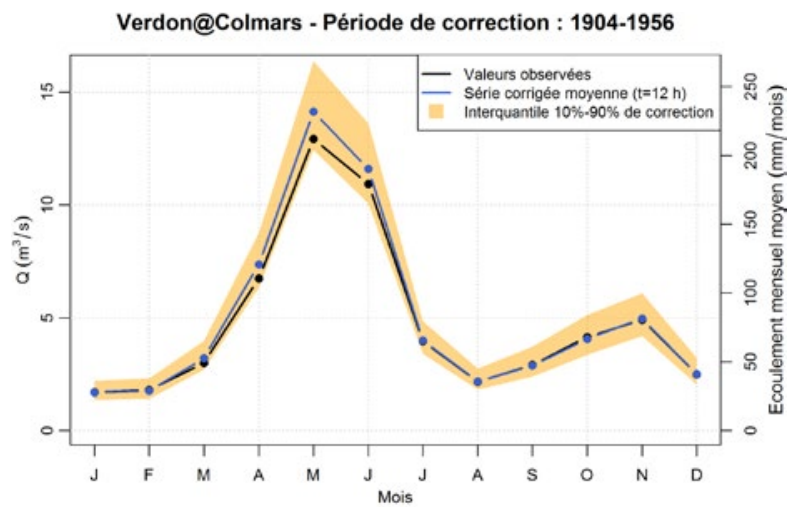

Figure 4 : Impact de la méthode de lecture des hauteurs sur le régime hydrologique du Verdon à Colmars.

\section{TEMOIGNAGES DE LA VARIABILITÉ HYDROLOGIQUE PASSÉE}

Les longues séries de débits ainsi retrouvées et numérisées au cours de nos travaux représentent un patrimoine de données exceptionnel, en tant que témoin direct de la variabilité hydrologique de la Durance au cours du siècle dernier, sur une diversité de témoins hydrologiques. Ces séries permettent ainsi de contextualiser et relativiser les études d'impacts de changement climatique, sachant ce qu'on a déjà observé depuis plus de 100 ans. Par exemple, la Figure 5 présente la variabilité du débit moyen annuel (normé par rapport au débit moyen 1900-2010) sur 22 stations de débits du projet R2D2. Ces séries de débits ont été obtenues par une méthode de simulation hydroclimatique développée par Kuentz et al. (2013) qui a été validée sur le passé uniquement grâce aux données anciennes que nous avons trouvé. Cette figure montre la relative homogénéité du signal hydrologique entre les bassins, malgré une différence de régimes hydrologiques. On y observe une forte variabilité hydrologique au cours des 100 dernières années et on retrouve ainsi très clairement des épisodes documentés de forte hydraulicité et de crues (1915-1920, 1935-1936, début 1960) ou des périodes de faible hydraulicité et d'étiages (1921, années 1940, fin des années 1970 et 1980, 2003-2005).

\section{CONCLUSIONS ET PERSPECTIVES}

Cet article présente un ensemble de travaux qui permettent de valoriser un large patrimoine d'informations et de données anciennes relatives à l'hydrologie et l'hydrométrie de la Durance depuis le début du $20^{\text {ème }}$ siècle. Ces recherches nous ont tout d'abord permis de témoigner de l'extraordinaire patrimoine de données sur ce bassin, où l'on retrouve entre autres une dizaine de séries séculaires de débit. Ensuite, concernant l'hydrométrie, ces travaux nous ont permis de nous rendre compte du fort avancement des connaissances dans ce domaine dès le début $\mathrm{du} 20^{\text {ème }}$ siècle, puisque la plupart des considérations de cette époque (incertitude des jaugeages, représentativité des courbes de tarage, stabilité de la section de mesure, transport solide, sécurité, etc.) restent encore et toujours

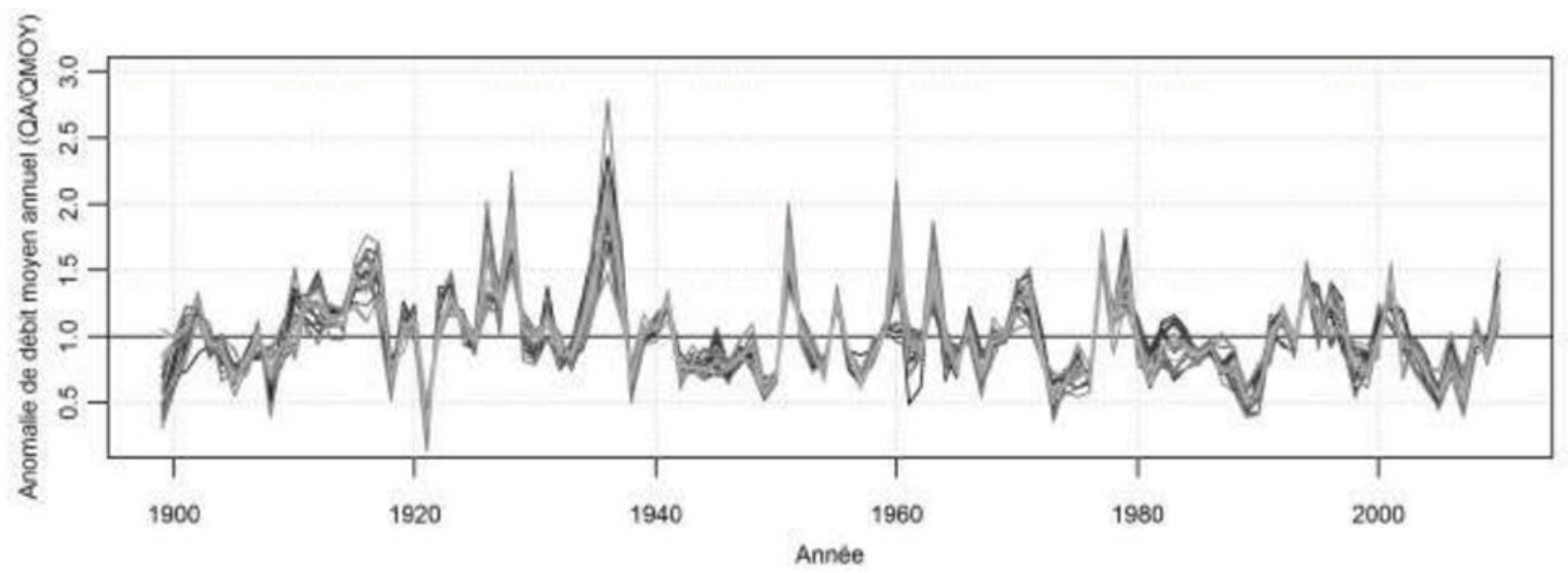

Figure 5 : Caractérisation de la variabilité du débit moyen annuel sur le bassin de la Durance (débits simulés sur la période 1900-2012 sur 22 bassins versants du projet R2D2). 
d'actualité. Enfin, bien qu'il reste encore beaucoup à faire pour caractériser la qualité et l'incertitude de ces données, nous avons pu mesurer que le mode d'échantillonnage des hauteurs pouvait sensiblement biaiser les débits des séries anciennes de bassins à régime nival.

Cet article permet également de montrer la richesse des longues séries d'observations et la chance de posséder de telles données pour contribuer aux débats sur les impacts du changement climatique. Actuellement, de nombreux travaux cherchent à caractériser la sensibilité des ressources en eau disponibles et de leurs usages aux scenarii climatiques du $21^{\text {ème }}$ siècle. Les données anciennes permettent de témoigner d'une certaine variabilité déjà observée à l'échelle du siècle dernier. Ces longues séries d'observations permettront ainsi de servir de références pour quantifier, relativiser ou rendre plus robuste la significativité des changements probables auxquels on peut s'attendre au cours de ce siècle.

\section{REFERENCES}

Céur D. (2012) — Enquête documentaire sur les données hydrologiques et hydrométriques anciennes du bassin de la Durance. Commanditaire : EDF-DTG. Rapport 1.29 p. et rapport 2. $33 p$

Kuentz A., Mathevet T., Gailhard, J ; Perret C., Andreassian V. (2013) - More than 100 years of climatic and hydrologic variability of a mediterranean and montainous watershed : the Durance river. Cold and Mountain Region Hydrological Systems Under Climate Change: Towards Improved Projections. Proceedings of H02, IAHS-IAPSO-IASPEI Assembly, Gothenburg, Sweden, July 2013, IAHS Publ. 360

IMBEAux E. (1892) - La Durance : régime, crues et inondations. Paris, France: Vve Ch. Dunod. 200 p

LALlEMENT C. (2002) - Quelques enseignements tirés de l'étude d'une longue série hydrométrique : la Durance au Pont de Mirabeau (1832-1959). DOI: 10.1051/lhb/2002058. La Houille Blanche. 4-5 : 51-55 